\title{
NULL-FINITE SETS IN TOPOLOGICAL GROUPS AND THEIR APPLICATIONS
}

\author{
TARAS BANAKH AND ELIZA JABŁOŃSKA
}

\begin{abstract}
In the paper we introduce and study a new family of "small" sets which is tightly connected with two well known $\sigma$-ideals: of Haar-null sets and of Haar-meager sets. We define a subset $A$ of a topological group $X$ to be null-finite if there exists a convergent sequence $\left(x_{n}\right)_{n \in \omega}$ in $X$ such that for every $x \in X$ the set $\left\{n \in \omega: x+x_{n} \in A\right\}$ is finite. We prove that each null-finite Borel set in a complete metric Abelian group is Haar-null and Haar-meager. The Borel restriction in the above result is essential as each non-discrete metric Abelian group is the union of two null-finite sets. Applying null-finite sets to the theory of functional equations and inequalities, we prove that a mid-point convex function $f: G \rightarrow \mathbb{R}$ defined on an open convex subset $G$ of a metric linear space $X$ is continuous if it is upper bounded on a subset $B$ which is not null-finite and whose closure is contained in $G$. This gives an alternative short proof of a known generalization of Bernstein-Doetsch theorem (saying that a mid-point convex function $f: G \rightarrow \mathbb{R}$ defined on an open convex subset $G$ of a metric linear space $X$ is continuous if it is upper bounded on a non-empty open subset $B$ of $G$ ). Since Borel Haar-finite sets are Haar-meager and Haar-null, we conclude that a mid-point convex function $f: G \rightarrow \mathbb{R}$ defined on an open convex subset $G$ of a complete linear metric space $X$ is continuous if it is upper bounded on a Borel subset $B \subset G$ which is not Haar-null or not Haar-meager in $X$. The last result resolves an old problem in the theory of functional equations and inequalities posed by Baron and Ger in 1983.
\end{abstract}

\section{INTRODUCTION}

In 1920 Steinhaus 29] proved that for any measurable sets $A, B$ of positive Haar measure in a locally compact Polish group $X$ the sum $A+B:=\{a+b: a \in A, b \in B\}$ has non-empty interior in $X$ and the difference $B-B:=\{a-b: a, b \in B\}$ is a neighborhood of zero in $X$.

In [8] Christensen extended the "difference" part of the Steinhaus results to all Polish Abelian groups proving that for a Borel subset $B$ of a Polish Abelian group $X$ the difference $B-B$ is a neighborhood of zero if $B$ is not Haar-null. Christensen defined a Borel subset $B \subset X$ to be Haar-null if there exists a Borel $\sigma$-additive probability measure $\mu$ on $X$ such that $\mu(B+x)=0$ for all $x \in X$.

A topological version of Steinhaus theorem was obtained by Pettis 25] and Piccard [26] who proved that for any non-meager Borel sets $A, B$ in a Polish group $X$ the sum $A+B$ has non-empty interior and the difference $B-B$ is a neighborhood of zero.

In 2013 Darji 9 introduced a subideal of the $\sigma$-ideal of meager sets in a Polish group, which is a topological analog to the $\sigma$-ideal of Haar-null sets. Darji defined a Borel subset $B$ of a Polish group $X$ to be Haar-meager if there exists a continuous map $f: K \rightarrow X$ from a non-empty compact metric space $K$ such that for every $x \in X$ the preimage $f^{-1}(B+x)$ is meager in $K$. By [18, for every Borel subset $B \subset X$ which is not Haar-meager in a Polish Abelian group $X$ the difference $B-B$ is a neighborhood of zero.

It should be mentioned that in contrast to the "difference" part of the Steinhaus and Piccard-Pettis theorems, the "additive" part can not be generalized to non-locally compact groups: by [23, 18] each non-locally compact Polish Abelian group $X$ contains two closed sets $A, B$ whose sum $A+B$ has empty interior but $A, B$ are neither Haar-null nor Haar-meager in $X$. In the Polish group $X=\mathbb{R}^{\omega}$, for such sets $A, B$ we can take the positive cone $\mathbb{R}_{+}^{\omega}$.

Steinhaus-type theorems has significant applications in the theory of functional equations and inequalities. For example, the "additive" part of Steinhaus Theorem can be applied to prove that a mid-point convex function $f: \mathbb{R}^{n} \rightarrow \mathbb{R}$ is continuous if it is upper bounded on some measurable set $B \subset \mathbb{R}^{n}$ of positive Lebesgue measure (see e.g. [20, p.210]). We recall that a function $f: G \rightarrow \mathbb{R}$ defined on a convex subset of a linear space is mid-point convex if $f\left(\frac{x+y}{2}\right) \leq \frac{f(x)+f(y)}{2}$ for any $x, y \in G$. Unfortunately, due to the example of Matoušková and Zelený 23 . we know that the "additive" part of the Steinhaus theorem does not extend to non-locally

1991 Mathematics Subject Classification. 39B52, 28C10, 18B30, 54E52.

Key words and phrases. Automatic continuity, additive function, mid-point convex function, null-finite set, Haar-null set, Haar-meager set. 
compact Polish Abelian groups. This leads to the following natural problem whose "Haar-null" version was posed by Baron and Ger in [3, P239].

Problem (Baron, Ger, 1983). Is the continuity of an additive or mid-point convex function $f: X \rightarrow \mathbb{R}$ on a Banach space $X$ equivalent to the upper boundedness of the function on some Borel subset $B \subset X$ which is not Haar-null or not Haar-meager?

In this paper we give the affirmative answer to the Baron-Ger Problem applying a new concept of a nullfinite set, which will be introduced in Section 2, In Section 3 we show that non-null-finite sets $A$ in metric groups $X$ possess a Steinhaus-like property: if a subset $A$ of a metric group $X$ is not null-finite, then $A-\bar{A}$ is a neighborhood of $\theta$ and for some finite set $F \subset X$ the set $F+(A-A)$ is a neighborhood of zero in $X$. In Sections 5 and 6 we shall prove that Borel null-finite sets in Polish Abelian groups are Haar-meager and Haar-null. On the other hand, in Example 7.1 we show that the product $\prod_{n \in \mathbb{N}} C_{n}$ of cyclic groups contains a closed Haar-null set which is not null-finite and in Example 7.2 we show that the product $\prod_{n \in \mathbb{N}} C_{2^{n}}$ contains a null-finite $G_{\delta}$-set which cannot be covered by countably many closed Haar-null sets. In Section 8 we prove that each non-discrete metric Abelian group is the union of two null-finite sets and also observe that sparse sets (in the sense of Lutsenko-Protasov [22]) are null-finite. In Section 9 we apply null-finite sets to prove that an additive functional $f: X \rightarrow \mathbb{R}$ on a Polish Abelian group is continuous if it is upper bounded on some subset $B \subset X$ which is not null-finite in $X$. In Section 10 we generalize this result to additive functions with values in Banach and locally convex spaces. Finally, in Section 11] we prove a generalization of Bernstein-Doetsch theorem and next apply it in the proof of a continuity criterion for mid-point convex functions on complete metric linear spaces, thus answering the Baron-Ger Problem. In Section 12 we pose some open problems related to null-finite sets.

\section{Preliminaries}

All groups considered in this paper are Abelian. The neutral element of a group will be denoted by $\theta$. For a group $G$ by $G^{*}$ we denote the set of non-zero elements in $G$. By $C_{n}=\left\{z \in \mathbb{C}: z^{n}=1\right\}$ we denote the cyclic group of order $n$.

By a (complete) metric group we understand an Abelian group $X$ endowed with a (complete) invariant metric $\|\cdot-\cdot\|$. The invariant metric $\|\cdot-\cdot\|$ determines (and can be recovered from) the prenorm $\|\cdot\|$ defined by $\|x\|:=\|x-\theta\|$. So, a metric group can be equivalently defined as a group endowed with a prenorm.

Formally, a prenorm on a group $X$ is a function $\|\cdot\|: X \rightarrow \mathbb{R}_{+}:=[0, \infty)$ satisfying three axioms:

- $\|x\|=0$ iff $x=\theta$;

- $\|-x\|=\|x\|$;

- $\|x+y\| \leq\|x\|+\|y\|$

for any $x, y \in X$; see [1, §3.3].

Each metric group is a topological group with respect to the topology, generated by the metric.

All linear spaces considered in this paper are over the field $\mathbb{R}$ of real numbers. By a metric linear space we understand a linear space endowed with an invariant metric.

A non-empty family $\mathcal{I}$ of subsets of a set $X$ is called ideal of sets on $X$ if $\mathcal{I}$ satisfies the following conditions:

- $X \notin \mathcal{I}$;

- for any subsets $J \subset I \subset X$ the inclusion $I \in \mathcal{I}$ implies $J \in \mathcal{I}$;

- for any sets $A, B \in \mathcal{I}$ we have $A \cup B \in \mathcal{I}$.

An ideal $\mathcal{I}$ on $X$ is called a $\sigma$-ideal if for any countable subfamily $\mathcal{C} \subset \mathcal{I}$ the union $\bigcup \mathcal{C}$ belongs to $X$.

An ideal $\mathcal{I}$ on a group $X$ is called invariant if for any $I \in \mathcal{I}$ and $x \in X$ the shift $x+I$ of $I$ belongs to the ideal $\mathcal{I}$.

For example, the family $[X]^{<\omega}$ of finite subsets of an infinite group $X$ is an invariant ideal on $X$.

A topological space is Polish if it is homeomorphic to a separable complete metric space. A topological space is analytic if it is a continuous image of a Polish space.

\section{INTRODUCING NULL-FINITE SETS IN TOPOLOGICAL GROUPS}

In this section we introduce the principal new notion of this paper.

A sequence $\left(x_{n}\right)_{n \in \omega}$ in a topological group $X$ is called a null-sequence if it converges to the neutral element $\theta$ of $X$. 
Definition 2.1. A set $A$ of a topological group $X$ is called null-finite if there exists a null-sequence $\left(x_{n}\right)_{n \in \omega}$ in $X$ such that for every $x \in X$ the set $\left\{n \in \omega: x+x_{n} \in A\right\}$ is finite.

Null-finite sets in metrizable topological groups can be defined as follows.

Proposition 2.2. For a non-empty subset $A$ of a metric group $X$ the following conditions are equivalent:

(1) A is null-finite;

(2) there exists an infinite compact set $K \subset X$ such that for every $x \in X$ the intersection $K \cap(x+A)$ is finite;

(3) there exists a continuous map $f: K \rightarrow X$ from an infinite compact space $K$ such that for every $x \in X$ the preimage $f^{-1}(A+x)$ is finite.

Proof. The implications (1) $\Rightarrow(2) \Rightarrow(3)$ are obvious, so it is enough to prove $(3) \Rightarrow(1)$. Assume that $f: K \rightarrow X$ is a continuous map from an infinite compact space $K$ such that for every $x \in X$ the preimage $f^{-1}(A+x)$ is finite. Fix any point $a \in A$. It follows that for every $x \in X$ the fiber $f^{-1}(a+x) \subset f^{-1}(A+x)$ is finite and hence the image $f(K)$ is infinite. So, we can choose a sequence $\left(y_{n}\right)_{n \in \omega}$ of pairwise distinct points in $f(K)$. Because of the compactness and metrizability of $f(K)$, we can additionally assume that the sequence $\left(y_{n}\right)_{n \in \omega}$ converges to some point $y_{\infty} \in f(K)$. Then the sequence $\left(x_{n}\right)_{n \in \omega}$ of points $x_{n}:=y_{n}-y_{\infty}, n \in \omega$, is a null-sequence witnessing that the set $A$ is null-finite.

Null-finite sets are "small" in the following sense.

Proposition 2.3. For any null-finite set $A$ in a metric group $X$ and any finite subset $F \subset X$ the set $F+A$ has empty interior in $X$.

Proof. Since $A$ is null-finite, there exists a null-sequence $\left(x_{n}\right)_{n \in \omega}$ in $X$ such that for every $x \in X$ the set $\left\{n \in \omega: x+x_{n} \in A\right\}$ is finite.

To derive a contradiction, assume that for some finite set $F$ the set $F+A$ has non-empty interior $U$ in $X$. Then for any point $u \in U$ the set $\left\{n \in \omega: u+x_{n} \in U\right\} \subset\left\{n \in \omega: u+x_{n} \in F+A\right\}$ is infinite. By the Pigeonhole Principle, for some $y \in F$ the set $\left\{n \in \omega: u+x_{n} \in y+A\right\}$ is infinite and so is the set $\left\{n \in \omega:-y+u+x_{n} \in A\right\}$. But this contradicts the choice of the sequence $\left(x_{n}\right)_{n \in \omega}$.

Now we present some examples of closed sets which are (or are not) null-finite. We recall that for a group $G$ by $G^{*}$ we denote the set $G \backslash\{\theta\}$ of non-zero elements of $G$.

Example 2.4. Let $\left(G_{n}\right)_{n \in \omega}$ be a sequence of finite groups. The set $A:=\prod_{n \in \omega} G_{n}^{*}$ is null-finite in the compact metrizable topological group $G=\prod_{n \in \omega} G_{n}$ if and only if $\lim _{n \rightarrow \infty}\left|G_{n}\right| \neq \infty$.

Proof. First, we assume that $\lim _{n \rightarrow \infty}\left|G_{n}\right|=\infty$ and show that the set $A$ is not null-finite in $G$. Given a null-sequence $\left(x_{n}\right)_{n \in \omega}$ in the compact topological group $G$, we should find an element $a \in G$ such that the set $\left\{n \in \omega: a+x_{n} \in A\right\}$ is infinite.

It will be convenient to think of elements of the group $G$ as functions $x: \omega \rightarrow \bigoplus_{n \in \omega} G_{n}$ such that $x(k) \in G_{k}$ for all $k \in \omega$.

Taking into account that $\lim _{k \rightarrow \infty}\left|G_{k}\right|=\infty$ and $\lim _{n \rightarrow \infty} x_{n}=\theta \in G$, we can inductively construct an increasing number sequence $\left(n_{k}\right)_{k \in \omega}$ such that $n_{0}=0$ and for every $k \in \mathbb{N}$ and $m \geq n_{k}$ the following two conditions are satisfied:

(1) $x_{m}(i)=\theta$ for all $i<n_{k-1}$;

(2) $\left|G_{m}\right| \geq k+3$.

We claim that for every $i \geq n_{1}$ the set $\left\{x_{n_{k}}(i): k \in \omega\right\}$ has cardinality $<\left|G_{i}\right|$. Indeed, given any $i \geq n_{1}$, we can find a unique $j \geq 1$ such that $n_{j} \leq i<n_{j+1}$ and conclude that $x_{n_{k}}(i)=\theta$ for all $k \geq j+1$ (by the condition (1)). Then the set $\left\{x_{n_{k}}(i): k \in \omega\right\}=\{\theta\} \cup\left\{x_{n_{k}}(i): k \leq j\right\}$ has cardinality $\leq 1+(j+1)<j+3 \leq\left|G_{i}\right|$. The last inequality follows from $i \geq n_{k}$ and condition (2). Therefore, the set $\left\{x_{n_{k}}(i): k \in \omega\right\}$ has cardinality $<\left|G_{i}\right|$ and we can choose a point $a_{i} \in G_{i} \backslash\left\{-x_{n_{k}}(i): k \in \omega\right\}$ and conclude that $a_{i}+x_{n_{k}}(i) \in G_{i}^{*}$ for all $k \in \omega$. Then the element $a=\left(a_{i}\right)_{i \in \omega} \in G$ has the required property as the set $\left\{n \in \omega: a+x_{n} \in A\right\} \supset\left\{n_{k}\right\}_{k \in \omega}$ is infinite.

Next, assuming that $\lim _{k \rightarrow \infty}\left|G_{k}\right| \neq \infty$, we shall prove that the set $A$ is Haar-finite in $G$. Since $\lim _{k \rightarrow \infty}\left|G_{k}\right| \neq$ $\infty$, for some $l \in \omega$ the set $\Lambda=\left\{k \in \omega:\left|G_{k}\right|=l\right\}$ is infinite.

Since $\lim _{n \rightarrow \infty}\left(1-\frac{1}{l}\right)^{n}=0$, there exists $n \in \mathbb{N}$ such that $\left(1-\frac{1}{l}\right)^{n}<\frac{1}{l}$ and hence $l(l-1)^{n}<l^{n}$. This implies that for every $k \in \Lambda$ the set $G_{k} \cdot\left(G_{k}^{*}\right)^{n}:=\left\{\left(x+a_{1}, \cdots x+a_{n}\right): x \in G_{k}, a_{1}, \ldots, a_{n} \in G_{k}^{*}\right\}$ has cardinality $\left|G_{k} \cdot\left(G_{k}^{*}\right)^{n}\right| \leq l(l-1)^{n}<l^{n}=\left|G_{k}^{n}\right|$. Consequently, the (compact) set $G \cdot A^{n}=\left\{\left(x+a_{1}, \ldots, x+a_{n}\right): x \in\right.$ 
$\left.G, a_{1}, \ldots, a_{n} \in A\right\}$ is nowhere dense in the compact topological group $G^{n}$. Now Theorem 2.5 ensures that $A$ is null-finite in $G$.

Theorem 2.5. A non-empty subset $A$ of a Polish group $X$ is null-finite in $X$ if for some $n \in \mathbb{N}$ the set $X \cdot A^{n}:=\left\{\left(x+a_{1}, \ldots, x+a_{n}\right): x \in X, a_{1}, \ldots, a_{n} \in A\right\}$ is meager in $X^{n}$.

Proof. Let $\mathcal{K}(X)$ be the space of non-empty compact subsets of $X$, endowed with the Vietoris topology. For a compact subset $K \subset X$ let $K_{*}^{n}=\left\{\left(x_{1}, \ldots, x_{n}\right) \in K^{n}:\left|\left\{x_{1}, \ldots, x_{n}\right\}\right|=n\right\}$ the set of $n$-tuples consisting of pairwise distinct points of $K$.

By Mycielski-Kuratowski Theorem [19, 19.1], the set $W_{*}(A):=\left\{K \in \mathcal{K}(X): K_{*}^{n} \cap\left(X \cdot A^{n}\right)=\emptyset\right\}$ is comeager in $\mathcal{K}(X)$ and hence contains some infinite compact set $K$. We claim that for every $x \in X$ the set $K \cap(x+A)$ has cardinality $<n$. Assuming the opposite, we could find $n$ pairwise distinct points $a_{1}, \ldots, a_{n} \in A$ such that $x+a_{i} \in K$ for all $i \leq n$. Then $\left(x+a_{1}, \ldots, x+a_{n}\right) \in K_{*}^{n} \cap X \cdot A^{n}$, which contradicts the inclusion $K \in W_{*}(A)$. By Proposition 2.2, the set $A$ is null-finite.

\section{A Steinhaus-like properties of Sets Which are not null-Finite}

In 29] Steinhaus proved that for a subset $A$ of positive Lebesgue measure in the real line, the set $A-A$ is a neighborhood of zero. In this section we establish three Steinhaus-like properties of sets which are not null-finite.

Theorem 3.1. If a subset $A$ of a metric group $X$ is not null-finite, then

(1) the set $A-\bar{A}$ is a neighborhood of $\theta$ in $X$;

(2) each neighborhood $U \subset X$ of $\theta$ contains a finite subset $F \subset U$ such that $F+(A-A)$ is a neighborhood of $\theta$.

(3) If $X$ is Polish (and $A$ is analytic), then $A-A$ is not meager in $X$ (and $(A-A)-(A-A)$ is a neighborhood of $\theta$ in $X)$.

Proof. 1. Assuming that $A-\bar{A}$ is not a neighborhood of $\theta$, we could find a null-sequence $\left(x_{n}\right)_{n \in \omega}$ contained in $X \backslash(A-\bar{A})$. Since $A$ is not null-finite, there exists $a \in X$ such that the set $\Omega=\left\{n \in \omega: a+x_{n} \in A\right\}$ is infinite. Then $a \in{\overline{\left\{a+x_{n}\right\}_{n \in \Omega}}}_{\bar{A}} \subset \bar{A}$ and hence $x_{n}=\left(a+x_{n}\right)-a \in A-\bar{A}$ for all $n \in \Omega$, which contradicts the choice of the sequence $\left(x_{n}\right)_{n \in \omega}$.

2. Fix a decreasing neighborhood base $\left(U_{n}\right)_{n \in \omega}$ at zero in $X$ such that $U_{0} \subset U$. For the proof by contradiction, suppose that for any finite set $F \subset U$ the set $F+(A-A)$ is not a neighborhood of zero. Then we can inductively construct a null-sequence $\left(x_{n}\right)_{n \in \omega}$ such that $x_{n} \in U_{n} \backslash \bigcup_{0 \leq i<n}\left(x_{i}+A-A\right)$ for all $n \in \omega$. Observe that for each $z \in X$ the set $\left\{n \in \omega: z+x_{n} \in A\right\}$ contains at most one point. Indeed, in the opposite case we could find two numbers $k<n$ with $z+x_{k}, z+x_{n} \in A$ and conclude that $z \in-x_{k}+A$ and hence $x_{n} \in-z+A \subset x_{k}-A+A$, which contradicts the choice of the number $x_{n}$. The sequence $\left(x_{n}\right)_{n \in \omega}$ witnesses that the set $A$ is null-finite in $X$.

3. Finally assume that the group $X$ is Polish. To derive a contradiction, assume that $A-A$ is meager. Since the map $\delta: X \times X \rightarrow X, \delta:(x, y) \mapsto x-y$, is open, the preimage $\delta^{-1}(A-A)$ is meager in $X \times X$. Observe that $\delta^{-1}(A-A)=X \cdot A^{2}$ where $X \cdot A^{2}=\{(x+a, x+b): x \in X, a, b \in A\}$. By Theorem 2.5, the set $A$ is null-finite, which is a desired contradiction. This contradiction shows that the set $A-A$ is not meager in $X$. If the space $A$ is analytic, then so is the subset $A-A$ of $X$. Being analytic, the set $A-A$ has the Baire Property in $X$; see [19, 29.14]. So, we can apply the Pettis-Piccard Theorem [19, 9.9] and conclude that $(A-A)+(A-A)$ is a neighborhood of $\theta$ in $X$.

The last statement of Theorem 3.1 does not hold without any assumptions on $A$ and $X$.

Example 3.2. Let $f: C_{2}^{\omega} \rightarrow C_{2}$ be a discontinuous homomorphism on the compact Polish group $X=C_{2}^{\omega}$ (thought as the vector space $C_{2}^{\omega}$ over the two-element field $\mathbb{Z} / 2 \mathbb{Z}$ ). Then the subgroup $A=f^{-1}(\theta)$ is not null-finite in $X$ (by Proposition 2.3), but $A-A=A=(A-A)-(A-A)$ is not a neighborhood of $\theta$ in $X$.

\section{A COMBINATORIAL CHARACTERIZATION OF NULl-Finite SETS IN COMPACT METRIC GROUPS}

In compact metric groups null-finite sets admit a purely combinatorial description.

Proposition 4.1. A non-empty subset $A$ of a compact metric group $X$ is null-finite if and only if there exists an infinite set $I \subset X$ such that for any infinite subset $J \subset I$ the intersection $\bigcap_{x \in J}(A-x)$ is empty. 
Proof. To prove the "only if" part, assume that $A \subset X$ is null-finite. So, there exists a null-sequence $\left(x_{n}\right)_{n \in \omega}$ such that for every $x \in X$ the set $\left\{n \in \omega: x+x_{n} \in A\right\}$ is finite. It follows that for every $x \in X$ the set $\left\{n \in \omega: x_{n}=x\right\}$ is finite and hence the set $I:=\left\{x_{n}\right\}_{n \in \omega}$ is infinite. We claim that this set has the required property. Indeed, assuming that for some infinite subset $J \subset I$ the intersection $\bigcap_{x \in J}(A-x)$ contains some point $a \in X$, we conclude that the set $\left\{n \in \omega: a+x_{n} \in A\right\}$ contains the set $\left\{n \in \omega: x_{n} \in J\right\}$ and hence is infinite, which contradicts the choice of the sequence $\left(x_{n}\right)_{n \in \omega}$.

To prove the "if" part, assume that there exists an infinite set $I \subset X$ such that for every infinite set $J \subset I$ the intersection $\bigcap_{x \in J}(A-x)$ is empty. By the compactness of the metric group $X$, some sequence $\left(x_{n}\right)_{n \in \omega}$ of pairwise distinct points of the infinite set $I$ converges to some point $x_{\infty} \in X$. Then the nullsequence $\left(z_{n}\right)_{n \in \omega}$ consisting of the points $z_{n}=x_{n}-x_{\infty}, n \in \omega$, witnesses that $A$ is null-finite. Assuming the opposite, we would find a point $a \in X$ such that the set $\left\{n \in \omega: a+z_{n} \in A\right\}$ is infinite. Then the set $J:=\left\{x \in I: a-x_{\infty}+x \in A\right\} \subset\left\{x_{n}: n \in \omega, a-x_{\infty}+x_{n} \in A\right\}$ is infinite, too, and the intersection $\bigcap_{x \in J}(A-x)$ contains the point $a-x_{\infty}$ and hence is not empty, which contradicts the choice of the set $I$.

Following Lutsenko and Protasov 22] we define a subset $A$ of an infinite group $X$ to be sparse if for any infinite set $I \subset X$ there exists a finite set $F \subset I$ such that $\bigcap_{x \in F}(x+A)$ is empty. By [22, Lemma 1.2] the family of sparse subsets of a group is an invariant ideal on $X$ (in contrast to the family of null-finite sets).

Proposition 4.2. Each sparse subset $A$ of a non-discrete metric group $X$ is null-finite.

Proof. Being non-discrete, the metric group $X$ contains a null-sequence $\left(x_{n}\right)_{n \in \omega}$ consisting of pairwise distinct points. Assuming that the sparse set $A$ is not null-finite, we can find a point $a \in X$ such that the set $\Omega:=\left\{n \in \omega: a+x_{n} \in A\right\}$ is infinite. Since $A$ is sparse, for the infinite set $I:=\left\{-x_{n}: n \in \Omega\right\}$ there exists a finite set $F \subset I$ such that the intersection $\bigcap_{x \in F}(x+A) \supset \bigcap_{m \in \Omega}\left(-x_{n}+A\right) \ni a$ is empty, which is not possible as this intersection contains the point $a$.

\section{Null-Finite Borel Sets ARe HaAR-MEAGer}

In this section we prove that each null-finite set with the universal Baire property in a complete metric group is Haar-meager.

A subset $A$ of a topological group $X$ is defined to have the universal Baire Property (briefly, $A$ is a $u B P$-set) if for any function $f: K \rightarrow X$ from a compact metrizable space $K$ the preimage $f^{-1}(A)$ has the Baire Property in $K$, which means that for some open set $U \subset K$ the symmetric difference $U \triangle f^{-1}(A)$ is meager in $K$. It is well-known that each Borel subset of a topological group has the universal Baire Property.

A uBP-set $A$ of a topological group $X$ is called Haar-meager if there exists a continuous map $f: K \rightarrow X$ from a compact metrizable space $K$ such that $f^{-1}(x+A)$ is meager in $K$ for all $x \in X$. By [9], for a complete metric group $X$ the family $\mathrm{HM}_{X}$ of subsets of Haar-meager uBP-sets in $X$ is an invariant $\sigma$-ideal on $X$. For more information on Haar-meager sets, see [9], [1], [10], 18].

Theorem 5.1. Each null-finite uBP-set in a compete metric group is Haar-meager.

Proof. To derive a contradiction, suppose that a null-finite $\mathrm{uBP}$-set $A$ in a complete metric group $(X,\|\cdot\|)$ is not Haar-meager. Since $A$ is null-finite, there exists a null-sequence $\left(a_{n}\right)_{n \in \omega}$ such that for every $x \in X$ the set $\left\{n \in \omega: x+a_{n} \in A\right\}$ is finite.

Replacing $\left(a_{n}\right)_{n \in \omega}$ by a suitable subsequence, we can assume that $\left\|a_{n}\right\|<\frac{1}{2^{n}}$ for all $n \in \omega$. For every $n \in \omega$ consider the compact set $K_{n}:=\{\theta\} \cup\left\{a_{m}\right\}_{m \geq n} \subset X$. The metric restriction on the sequence $\left(a_{n}\right)_{n \in \omega}$ implies that the function

$$
\Sigma: \prod_{n \in \omega} K_{n} \rightarrow X, \quad \Sigma:\left(x_{n}\right)_{n \in \omega} \mapsto \sum_{n \in \omega} x_{n},
$$

is well-defined and continuous (the proof of this fact can be found in [18; see the proof of Theorem 2).

Since the set $A$ is not Haar-meager and has $\mathrm{uBP}$, there exists a point $z \in X$ such that $B:=\Sigma^{-1}(z+A)$ is not meager and has the Baire Property in the compact metrizable space $K:=\prod_{n \in \omega} K_{n}$. Consequently, there exits a non-empty open set $U \subset K$ such that $U \cap B$ is comeager in $U$. Replacing $U$ by a smaller subset, we can assume that $U$ is of basic form $\{b\} \times \prod_{m>j} K_{m}$ for some $j \in \omega$ and some element $b \in \prod_{m<j} K_{m}$. It follows that for every $x \in K_{j} \backslash\{\theta\}$ the set $\{b\} \times\{x\} \times \prod_{m>j} K_{m}$ is closed and open in $K$ and hence the set $C_{x}:=\left\{y \in \prod_{m>j} K_{m}:\{b\} \times\{x\} \times\{y\} \subset U \cap B\right\}$ is comeager in $\prod_{m>j} K_{m}$. Then the intersection $\bigcap_{x \in K_{n} \backslash\{\theta\}} C_{x}$ is comeager and hence contains some point $c$. For this point we get the inclusion $\{b\} \times\left(K_{j} \backslash\{\theta\}\right) \times\{c\} \subset B$. Let $v \in K$ be a unique point such that $\{v\}=\{b\} \times\{\theta\} \times\{c\}$. For any $m \geq j$ the point $a_{m}$ belongs to $K_{j}$ and 
the inclusion $\{b\} \times\left\{a_{m}\right\} \times\{c\} \subset B$ implies that $\Sigma(v)+a_{m} \in z+A$. Consequently, the element $u:=-z+\Sigma(v)$ has the property $u+a_{m} \in A$ for all $m \geq j$, which implies that the set $\left\{n \in \omega: u+a_{n} \in A\right\}$ is infinite. But this contradicts the choice of the sequence $\left(a_{n}\right)_{n \in \omega}$.

\section{Null-Finite Borel Sets ARe HaAR-Null}

In this section we prove that each universally measurable null-finite set in a complete metric group is Haarnull.

A subset $A$ of a topological group $X$ is defined to be universally measurable if $A$ is measurable with respect to any $\sigma$-additive Radon Borel probability measure on $X$. It is clear that each Radon Borel subset of a topological group is universally measurable. We recall that a measure $\mu$ on a topological space $X$ is Radon if for any $\varepsilon>0$ there exists a compact subset $K \subset X$ such that $\mu(X \backslash K)<\varepsilon$.

A universally measurable subset $A$ of a topological group $X$ is called Haar-null if there exists a $\sigma$-additive Radon Borel probability measure $\mu$ on $X$ such that $\mu(x+A)=0$ for all $x \in X$. By [8, for any complete metric group $X$ the family $\mathrm{HN}_{X}$ of subsets of universally measurable Haar-null subsets of $X$ is an invariant $\sigma$-ideal in $X$. For more information on Haar-null sets, see [8], [16, [17] and also e.g. [13], [15, [23, [28].

Theorem 6.1. Each universally measurable null-finite set $A$ in a complete metric group $X$ is Haar-null.

Proof. To derive a contradiction, suppose that the null-finite set $A$ is not Haar-null in $X$. Since $A$ is null-finite, there exists a null-sequence $\left(a_{n}\right)_{n \in \omega}$ such that for each $x \in X$ the set $\left\{n \in \omega: x+a_{n} \in A\right\}$ is finite. Replacing $\left(a_{n}\right)_{n \in \omega}$ by a suitable subsequence, we can assume that $\left\|a_{n}\right\| \leq \frac{1}{4^{n}}$ for all $n \in \omega$.

Consider the compact space $\Pi:=\prod_{n \in \omega}\left\{0,1, \ldots, 2^{n}\right\}$ endowed with the product measure $\lambda$ of uniformly distributed measures on the finite discrete spaces $\left\{0,1,2,3, \ldots, 2^{n}\right\}, n \in \omega$. Consider the map

$$
\Sigma: \Pi \rightarrow X, \quad \Sigma:\left(p_{i}\right)_{i \in \omega} \mapsto \sum_{i=0}^{\infty} p_{i} a_{i} .
$$

Since $\left\|p_{i} a_{i}\right\| \leq 2^{i}\left\|a_{i}\right\| \leq 2^{i} \frac{1}{4^{i}}=\frac{1}{2^{i}}$, the series $\sum_{i=0}^{\infty} p_{i} a_{i}$ is convergent and the function $\Sigma$ is well-defined and continuous.

Since the set $A$ is not Haar-null, there exists an element $z \in X$ such that the preimage $\Sigma^{-1}(z+A)$ has positive $\lambda$-measure and hence contains a compact subset $K$ of positive measure. For every $n \in \omega$ consider the subcube $\Pi_{n}:=\prod_{i=0}^{n-1}\left\{0,1,2, \ldots, 2^{i}\right\} \times \prod_{i=n}^{\infty}\left\{1,2, \ldots, 2^{i}\right\}$ of $\Pi$ and observe that $\lambda\left(\Pi_{n}\right) \rightarrow 1$ as $n \rightarrow \infty$. Replacing $K$ by $K \cap \Pi_{l}$ for a sufficiently large $l$, we can assume that $K \subset \Pi_{l}$. For every $m \geq l$ let $s_{m}: \Pi_{l} \rightarrow \Pi$ be the "back-shift" defined by the formula $s_{m}\left(\left(x_{i}\right)_{i \in \omega}\right):=\left(y_{i}\right)_{i \in \omega}$ where $y_{i}=x_{i}$ for $i \neq m$ and $y_{i}=x_{i}-1$ for $i=m$.

Claim 6.2. For any compact set $C \subset \Pi_{l}$ of positive measure $\lambda(C)$ and any $\varepsilon>0$ there exists $k \geq l$ such that for any $m \geq k$ the intersection $C \cap s_{m}(C)$ has measure $\lambda\left(C \cap s_{m}(C)\right)>(1-\varepsilon) \lambda(C)$.

Proof. By the regularity of the measure $\lambda$, the set $C$ has a neighborhood $O(C) \subset \Pi$ such that $\lambda(O(C) \backslash C)<$ $\varepsilon \lambda(K)$. By the compactness of $C$, there exists $k \geq l$ such that for any $m \geq k$ the shift $s_{m}(C)$ is contained in $O(C)$. Hence $\lambda\left(s_{m}(C) \backslash C\right) \leq \lambda(O(C) \backslash C)<\varepsilon \lambda(C)$ and thus $\lambda\left(s_{m}(C) \cap C\right)=\lambda\left(s_{m}(C)\right)-\lambda\left(s_{m}(C) \backslash C\right)>$ $\lambda(C)-\varepsilon \lambda(C)=(1-\varepsilon) \lambda(C)$.

Using Claim 6.2 we can choose an increasing number sequence $\left(m_{k}\right)_{k \in \omega}$ such that $m_{0}>l$ and the set $K_{\infty}:=\bigcap_{k \in \omega} s_{m_{k}}(K)$ has positive measure and hence contains a point $\vec{b}:=\left(b_{i}\right)_{i \in \omega}$. It follows that for every $k \in \omega$ the point $\vec{b}_{k}:=s_{m_{k}}^{-1}(\vec{b})$ belongs to $K \subset \Sigma^{-1}(z+A)$.

Observe that $\Sigma\left(\vec{b}_{k}\right)=\Sigma(\vec{b})+a_{m_{k}} \in z+A$ and hence $-z+\Sigma(\vec{b})+a_{m_{k}} \in A$ for all $k \in \omega$, which contradicts the choice of the sequence $\left(a_{n}\right)_{n \in \omega}$.

Remark 6.3. After writing the initial version of this paper we discovered that a result similar to Theorem 6.1 was independently found by Bingham and Ostaszewski [6, Theorem 3].

\section{The $\sigma$-IDEAl Generated By (Closed) Borel null-Finite Sets}

In this section we introduce two new invariant $\sigma$-ideals generated by (closed) Borel null-finite sets and study the relation of these new ideals to the $\sigma$-ideals of Haar-null and Haar-meager sets.

Namely, for a complete metric group $X$ let

- $\sigma \mathrm{NF}_{X}$ be the smallest $\sigma$-ideal containing all Borel null-finite sets in $X$; 
- $\sigma \overline{\mathrm{NF}}_{X}$ be the smallest $\sigma$-ideal containing all closed null-finite sets in $X$;

- $\sigma \overline{\mathrm{HN}}_{X}$ be the smallest $\sigma$-ideal containing all closed Haar-null sets in $X$.

Theorems 5.1 and 6.1 imply that $\sigma \overline{\mathrm{NF}}_{X} \subset \sigma \overline{\mathrm{HN}}_{X}$ and $\sigma \mathrm{NF}_{X} \subset \mathrm{HN}_{X} \cap \mathrm{HM}_{X}$. So, we obtain the following diagram in which an arrow $\mathcal{A} \rightarrow \mathcal{B}$ indicates that $\mathcal{A} \subset \mathcal{B}$.

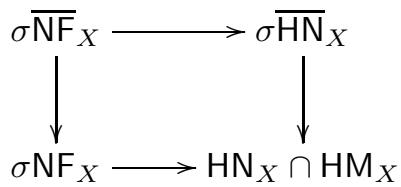

In Examples 7.1] and 7.2 we show that the $\sigma$-ideal $\sigma \overline{\mathrm{NF}}_{X}$ is strictly smaller than $\sigma \overline{\mathrm{HN}}_{X}$ and the ideal $\sigma \mathrm{NF}_{X}$ is not contained in $\sigma \overline{\mathrm{HN}}_{X}$.

Example 7.1. The closed set $A=\prod_{n \geq 2} C_{n}^{*}$ in the product $X=\prod_{n \geq 2} C_{n}$ of cyclic groups is Haar-null but cannot be covered by countably many closed null-finite sets. Consequently, $A \in \sigma \overline{\mathrm{HN}}_{X} \backslash \sigma \overline{\mathrm{NF}}_{X}$.

Proof. The set $A=\prod_{n=2}^{\infty} C_{n}^{*}$ has Haar measure

$$
\prod_{n=2}^{\infty} \frac{\left|C_{n}^{*}\right|}{\left|C_{n}\right|}=\prod_{n=2}^{\infty} \frac{n-1}{n}=0
$$

and hence is Haar-null in the compact Polish group $X$.

Next, we show that $A$ cannot be covered by countably many closed null-finite sets. To derive a contradiction, assume that $A=\bigcup_{n \in \omega} A_{n}$ where each $A_{n}$ is closed and null-finite in $X$. By the Baire Theorem, for some $n \in \omega$ the set $A_{n}$ has non-empty interior in $A$. Consequently, we can find $m>2$ and $a \in \prod_{n=2}^{m-1} C_{n}^{*}$ such that $\{a\} \times \prod_{n=m}^{\infty} C_{n}^{*} \subset A_{n}$. By Example 2.4 the set $\prod_{n=m}^{\infty} C_{n}^{*}$ is not null-finite in $\prod_{n=m}^{\infty} C_{n}$, which implies that the set $A_{n} \supset\{a\} \times \prod_{n=m}^{\infty} C_{n}^{*}$ is not null-finite in the group $X$. But this contradicts the choice of $A_{n}$.

Our next example shows that $\sigma \mathrm{NF}_{X} \not \subset \sigma \overline{\mathrm{HN}}_{X}$ for some compact Polish group $X$.

Example 7.2. For any function $f: \omega \rightarrow[2, \infty)$ with $\prod_{n \in \omega} \frac{f(n)-1}{f(n)}>0$, the compact metrizable group $X=$ $\prod_{n \in \mathbb{N}} C_{f(n)}$ contains a null-finite $G_{\delta}$-set $A \subset X$ which cannot be covered by countably many closed Haar-null sets in $X$. Consequently, $A \in \mathrm{NF}_{X} \backslash \sigma \overline{\mathrm{HN}}_{X}$.

Proof. For every $n \in \omega$ let $g_{n}$ be a generator of the cyclic group $C_{f(n)}$. In the compact metrizable group $X=\prod_{n \in \omega} C_{f(n)}$ consider the null-sequence $\left(x_{n}\right)_{n \in \omega}$ defined by the formula

$$
x_{n}(i)= \begin{cases}\theta & \text { if } i \leq n \\ g_{i} & \text { if } i>n .\end{cases}
$$

Consider the closed subset $B=\prod_{n \in \omega} C_{f(n)}^{*}$ in $X$ and observe that it has positive Haar measure, equal to the infinite product

$$
\prod_{n \in \omega} \frac{f(n)-1}{f(n)}>0
$$

It is easy to see that for every $n \in \omega$ the set $C_{f(n)}^{*}=C_{f(n)} \backslash\{\theta\}$ is not equal to $C_{f(n)}^{*} \cap\left(g_{n}+C_{f(n)}^{*}\right)$, which implies that the intersection $B \cap\left(x_{n}+B\right)$ is nowhere dense in $B$. Consequently, the set $A=B \backslash \bigcup_{n \in \omega}\left(x_{n}+B\right)$ is a dense $G_{\delta}$-set in $B$.

We claim that the set $A$ is null-finite. Given any $a \in X$ we should prove that the set $\left\{n \in \omega: a+x_{n} \in A\right\}$ is finite. If $a \notin B$, then the open set $X \backslash B$ is a neighborhood of $a$ in $X$. Since the sequence $\left(a+x_{n}\right)_{n \in \omega}$ converges to $a \in X \backslash B$, the set $\left\{n \in \omega: a+x_{n} \in B\right\} \supset\left\{n \in \omega: a+x_{n} \in A\right\}$ is finite.

If $a \in B$, then $\left\{n \in \omega: a+x_{n} \in A\right\} \subset\left\{n \in \omega:\left(B+x_{n}\right) \cap A \neq \emptyset\right\}=\emptyset$ by the definition of the set $A$.

Next, we prove that the $G_{\delta}$-set $A$ cannot be covered by countably many closed Haar-null sets. To derive a contradiction, assume that $A \subset \bigcup_{n \in \omega} F_{n}$ for some closed Haar-null sets $F_{n} \subset X$. Since the space $A$ is Polish, we can apply the Baire Theorem and find $n \in \omega$ such that the set $A \cap F_{n}$ has non-empty interior in $A$ and hence its closure $\overline{A \cap F_{n}}$ has non-empty interior in $B=\prod_{n \in \omega} C_{f(n)}^{*}$. It is easy to see that each non-empty open subset of $B$ has positive Haar measure in $X$. Consequently, the set $\overline{A \cap F_{n}}$ has positive Haar measure, which is not possible as this set is contained in the Haar-null set $F_{n}$. 
Remark 7.3. Answering a question posed in a preceding version of this paper, Adam Kwela 21 constructed two compact null-finite subsets $A, B$ on the real line, whose union $A \cup B$ is not null-finite. This means that the family of subsets of (closed) Borel null-finite subsets on the real line is not an ideal, and the ideal $\sigma \overline{\mathrm{NF}}_{\mathbb{R}}$ contains compact subsets of the real line, which fail to be null-finite.

\section{DeCOMPosing NON-DiscRete METRIC GROUPS INTO UNIONS OF TWO NULL-FINITE SETS}

By Theorem 6.1 and the countable additivity of the family of Borel Haar-null sets [8], the countable union of Borel null-finite sets in a complete metric group $X$ is Haar-null in $X$ and hence is not equal to $X$. So, a complete metric group cannot be covered by countably many Borel null-finite sets. This result dramatically fails for non-Borel null-finite sets.

Theorem 8.1. Each non-discrete metric group $X$ can be written as the union $X=A \cup B$ of two null-finite subsets $A, B$ of $X$.

Proof. Being non-discrete, the metric group $X$ contains a non-trivial null-sequence, which generates a nondiscrete countable subgroup $Z$ in $X$.

Let $Z=\left\{z_{n}\right\}_{n \in \omega}$ be an enumeration of the countable infinite group $Z$ such that $z_{0}=\theta$ and $z_{n} \neq z_{m}$ for any distinct numbers $n, m \in \omega$. By induction we can construct sequences $\left(u_{n}\right)_{n \in \omega}$ and $\left(v_{n}\right)_{n \in \omega}$ in $Z$ such that $u_{0}=v_{0}=\theta$ and for every $n \in \mathbb{N}$ the following two conditions are satisfied:

(1) $\left\|u_{n}\right\| \leq \frac{1}{n}$ and $u_{n} \notin\left\{-z_{i}+z_{j}+v_{k}: i, j \leq n, k<n\right\} \cup\left\{-z_{i}+z_{j}+u_{k}: i, j \leq n, k<n\right\}$;

(2) $\left\|v_{n}\right\| \leq \frac{1}{n}$ and $v_{n} \notin\left\{-z_{i}+z_{j}+u_{k}: i, j \leq n, k \leq n\right\} \cup\left\{-z_{i}+z_{j}+v_{k}: i, j \leq n, k<n\right\}$.

At the $n$-th step of the inductive construction, the choice of the points $u_{n}, v_{n}$ is always possible as the ball $\left\{z \in Z:\|z\| \leq \frac{1}{n}\right\}$ is infinite and $u_{n}, v_{n}$ should avoid finite sets.

After completing the inductive construction, we obtain the null-sequences $\left(u_{n}\right)_{n \in \omega}$ and $\left(v_{n}\right)_{n \in \omega}$ of pairwise distinct points of $Z$ such that for any points $x, y \in Z$ the intersection $\left\{x+u_{n}\right\}_{n \in \omega} \cap\left\{y+u_{m}\right\}_{m \in \omega}$ is finite and for every distinct points $x, y \in Z$ the intersections $\left\{x+u_{n}\right\}_{n \in \omega} \cap\left\{y+u_{m}\right\}_{m \in \omega}$ and $\left\{x+v_{n}\right\}_{n \in \omega} \cap\left\{y+v_{m}\right\}_{m \in \omega}$ are finite.

Using these facts, for every $n \in \omega$ we can choose a number $i_{n} \in \omega$ such that the set $\left\{z_{n}+v_{k}: k \geq i_{n}\right\}$ is disjoint with the set $\left\{z_{i}+v_{m}: i<n, m \in \omega\right\} \cup\left\{z_{i}+u_{m}: i \leq n, m \in \omega\right\}$ and the set $\left\{z_{n}+u_{k}: k \geq i_{n}\right\}$ is disjoint with the set $\left\{z_{i}+u_{m}: i<n, m \in \omega\right\} \cup\left\{z_{i}+v_{m}: i \leq n, m \in \omega\right\}$.

We claim that the set $A:=\bigcup_{n \in \omega}\left\{z_{n}+u_{m}: m>i_{n}\right\}$ is null-finite in $Z$. This will follow as soon as we verify the condition:

(3) for any $z \in Z$ the set $\left\{n \in \omega: z+v_{n} \in A\right\}$ is finite.

Find $j \in \omega$ such that $z=z_{j}$ and observe that for every $n \geq j$ the choice of the number $i_{n}$ guarantees that $\left\{z_{n}+u_{m}\right\}_{m>i_{n}} \cap\left\{z_{j}+v_{n}\right\}_{n \in \omega}=\emptyset$, so $\left\{n \in \omega: z+v_{n} \in A\right\}$ is contained in the finite set $\bigcup_{n<j}\left\{z_{n}+v_{m}\right\}_{m \in \omega} \cap$ $\left\{z_{j}+u_{m}\right\}_{m \in \omega}$ and hence is finite.

Next, we show that the set $B:=Z \backslash A$ is null-finite in $Z$. This will follow as soon as we verify the condition

(4) for any $z \in Z$ the set $\left\{m \in \omega: z+u_{m} \in B\right\}$ is finite.

Find a number $n \in \omega$ such that $z=z_{n}$ and observe that $\left\{m \in \omega: z+u_{m} \in B\right\}=\left\{m \in \omega: z_{n}+u_{m} \notin A\right\} \subset$ $\left\{m \in \omega: m \leq i_{n}\right\}$ is finite. Therefore, the sets $A$ and $B=Z \backslash A$ are null-finite in $Z$.

Using Axiom of Choice, choose a subset $S \subset X$ such that for every $x \in X$ the intersection $S \cap(x+Z)$ is a singleton. It follows that $X=S+Z=(S+A) \cup(S+B)$. We claim that the sets $S+A$ and $S+B$ are null-finite in $X$. This will follow as soon as for any $x \in X$ we check that the sets $\left\{n \in \omega: x+v_{n} \in S+A\right\}$ and $\left\{n \in \omega: x+u_{n} \in S+B\right\}$ are finite. Since $X=S+Z$, there exist elements $s \in S$ and $z \in Z$ such that $x=s+z$. Observe that if for $n \in \omega$ we get $x+v_{n} \in S+A$, then $s+z+v_{n}=t+a$ for some $t \in S$ and $a \in A \subset Z$. Subtracting $a$ from both sides, we get $t=s+z+v_{n}-a \in s+Z$ and hence $t=s$ as $S \cap(s+Z)$ is a singleton containing both points $t$ and $s$. So, the inclusion $x+v_{n}=s+z+v_{n} \in S+A$ is equivalent to $z+v_{n} \in A$. By analogy we can prove that $x+u_{n} \in S+B$ is equivalent to $z+u_{n} \in B$. Then the sets

$$
\left\{n \in \omega: x+v_{n} \in S+A\right\}=\left\{n \in \omega: z+v_{n} \in A\right\} \text { and }\left\{n \in \omega: x+u_{n} \in S+B\right\}=\left\{n \in \omega: z+u_{n} \in B\right\}
$$

are finite by the properties (3) and (4) of the null-finite sets $A, B$.

Proposition 2.3 and Theorem 8.1 imply the following corollary.

Corollary 8.2. Let $X$ be a non-discrete metric group. 
(1) Each null-finite subset of $X$ is contained in some invariant ideal on $X$;

(2) For any ideal $\mathcal{I}$ on $X$ there exists a null-finite set $A \subset X$ such that $A \notin \mathcal{I}$.

Proof. 1. For any null-finite set $A$ in $X$ the family

$$
\mathcal{I}_{A}=\left\{I \subset X: \exists F \in[X]^{<\omega} I \subset F A\right\}
$$

is an invariant ideal on $X$ whose elements have empty interiors in $X$ by Proposition 2.3 . We recall that by $[X]^{<\omega}$ we denote the ideal of finite subsets of $X$.

2. By Theorem 8.1 the group $X$ can be written as the union $X=A \cup B$ of two null-finite sets. Then for any ideal $\mathcal{I}$ on $X$ one of the sets $A$ or $B$ does not belong to $\mathcal{I}$.

\section{Applying NUll-Finite Sets to additive functionals}

In this section we apply null-finite sets to prove a criterion of continuity of additive functionals on metric groups.

A function $f: X \rightarrow Y$ between groups is called additive if $f(x+y)=f(x)+f(y)$ for every $x, y \in X$. An additive function into the real line is called an additive functional.

Theorem 9.1. An additive functional $f: X \rightarrow \mathbb{R}$ on a metric group $X$ is continuous if it is upper bounded on a set $B \subset X$ which is not null-finite.

Proof. Suppose that the functional $f$ is not continuous. Then there exists $\varepsilon>0$ such that $f(U) \not \subset(-\varepsilon, \varepsilon)$ for each neighborhood $U \subset X$ of zero. It follows that for every $n \in \omega$ there is a point $x_{n} \in X$ such that $\left\|x_{n}\right\| \leq \frac{1}{4^{n}}$ and $\left|f\left(x_{n}\right)\right|>\varepsilon$. Observe that, $\left\|2^{n} x_{n}\right\| \leq 2^{n} \cdot\left\|x_{n}\right\| \leq \frac{1}{2^{n}}$ and $\left|f\left(2^{n} x_{n}\right)\right|=2^{n}\left|f\left(x_{n}\right)\right|>2^{n} \varepsilon$. Choose $\varepsilon_{n} \in\{1,-1\}$ such that $\varepsilon_{n} f\left(2^{n} x_{n}\right)$ is positive and put $z_{n}:=\varepsilon_{n} 2^{n} x_{n}$. Then $f\left(z_{n}\right)>2^{n} \varepsilon$ and $\left\|z_{n}\right\| \leq \frac{1}{2^{n}}$.

We claim that the null-sequence $\left(z_{n}\right)_{n \in \omega}$ witnesses that the set $B$ is null-finite. Given any point $x \in X$ we need to check that the set $\Omega:=\left\{n \in \omega: x+z_{n} \in B\right\}$ is finite.

Let $M:=\sup f(B)$ and observe that for every $n \in \Omega$ we have

$$
M \geq f\left(x+z_{n}\right)=f(x)+f\left(z_{n}\right) \geq f(x)+2^{n} \varepsilon,
$$

which implies $2^{n} \leq \frac{1}{\varepsilon}(M-f(x))$. Hence the set $\Omega$ is finite. Therefore, the null-sequence $\left(z_{n}\right)_{n \in \omega}$ witnesses that the set $B$ is null-finite, which contradicts our assumption.

Theorems 9.1 and 5.1 imply

Corollary 9.2. An additive functional $f: X \rightarrow \mathbb{R}$ on a complete metric group is continuous if and only if it is upper bounded on some $u B P$ set $B \subset X$ which is not Haar-meager.

An analogous result for Haar-null sets follows from Theorems 9.1 and 6.1.

Corollary 9.3. An additive functional $f: X \rightarrow \mathbb{R}$ on a complete metric group is continuous if and only if it is upper bounded on some universally measurable set $B \subset X$ which is not Haar-null.

\section{Applying NUlL-Finite SETS to AdDitive FunCtions}

In this section we prove some continuity criteria for additive functions with values in Banach spaces or locally convex spaces.

Corollary 10.1. An additive function $f: X \rightarrow Y$ from a complete metric group $X$ to a Banach space $Y$ is continuous if for any linear continuous functional $y^{*}: Y \rightarrow \mathbb{R}$ the function $y^{*} \circ f: X \rightarrow \mathbb{R}$ is upper bounded on some set $B \subset X$ which is not null-finite in $X$.

Proof. Assuming that $f$ is discontinuous, we can find $\varepsilon>0$ such that for each neighborhood $U \subset X$ of $\theta$ we get $f(U) \not \subset B_{\varepsilon}:=\{y \in Y:\|y\|<\varepsilon\}$. Then for every $n \in \omega$ we can find a point $x_{n} \in X$ such that $\left\|x_{n}\right\| \leq \frac{1}{4^{n}}$ and $\left\|f\left(x_{n}\right)\right\| \geq \varepsilon$. Since $\left\|2^{n} x_{n}\right\| \leq 2^{n}\left\|x_{n}\right\| \leq \frac{1}{2^{n}}$, the sequence $\left(2^{n} x_{n}\right)_{n \in \omega}$ is a null-sequence in $X$. On the other hand, $\left\|f\left(2^{n} x_{n}\right)\right\|=2^{n}\left\|f\left(x_{n}\right)\right\| \geq 2^{n} \varepsilon$ for all $n \in \omega$, which implies that the set $\left\{f\left(2^{n} x_{n}\right)\right\}_{n \in \omega}$ is unbounded in the Banach space $Y$. By the Banach-Steinhaus Uniform Boundedness Principle [14, 3.15], there exists a linear continuous functional $y^{*}: Y \rightarrow \mathbb{R}$ such that the set $\left\{y^{*} \circ f\left(2^{n} x_{n}\right): n \in \omega\right\}$ is unbounded in $\mathbb{R}$. By our assumption, the additive functional $y^{*} \circ f: X \rightarrow \mathbb{R}$ is upper bounded on some set $B \subset X$ which is not null-finite in $X$. By Theorem 9.1, the additive functional $y^{*} \circ f$ is continuous and thus the sequence $\left(y^{*} \circ f\left(2^{n} x_{n}\right)\right)_{n \in \omega}$ converges to zero and hence cannot be unbounded in $\mathbb{R}$. This is a desired contradiction, completing the proof. 
Corollary 10.1 admits a self-generalization.

Theorem 10.2. An additive function $f: X \rightarrow Y$ from a complete metric group $X$ to a locally convex space $Y$ is continuous if for any linear continuous functional $y^{*}: Y \rightarrow \mathbb{R}$ the function $y^{*} \circ f: X \rightarrow \mathbb{R}$ is upper bounded on some set $B \subset X$ which is not null-finite in $X$.

Proof. This theorem follows from Corollary 10.1 and the well-known fact [27, p.54] that each locally convex space is topologically isomorphic to a linear subspace of a Tychonoff product of Banach spaces.

Combining Theorem 10.2 with Theorems 5.1 and 6.1, we derive two corollaries.

Corollary 10.3. An additive function $f: X \rightarrow Y$ from a complete metric group $X$ to a locally convex space $Y$ is continuous if for any linear continuous functional $y^{*}: Y \rightarrow \mathbb{R}$ the function $y^{*} \circ f: X \rightarrow \mathbb{R}$ is upper bounded on some $u B P$-set $B \subset X$ which is not Haar-meager in $X$.

Corollary 10.4. An additive function $f: X \rightarrow Y$ from a complete metric group $X$ to a locally convex space $Y$ is continuous if for any linear continuous functional $y^{*}: Y \rightarrow \mathbb{R}$ the function $y^{*} \circ f: X \rightarrow \mathbb{R}$ is upper bounded on some universally measurable set $B \subset X$ which is not Haar-null in $X$.

\section{Applying NUlL-Finite Sets to Mid-Point CONVEX FUnCtions}

In this section we apply null-finite sets to establish a continuity criterion for mid-point convex functions on linear metric spaces.

A function $f: C \rightarrow \mathbb{R}$ on a convex subset $C$ of a linear space is called mid-point convex if

$$
f\left(\frac{x}{2}+\frac{y}{2}\right) \leq \frac{1}{2} f(x)+\frac{1}{2} f(y)
$$

for any $x, y \in C$. Mid-point convex functions are alternatively called Jensen convex.

Theorem 11.1. A mid-point convex function $f: G \rightarrow \mathbb{R}$ defined on an open convex subset $G \subset X$ of a metric linear space $X$ is continuous if and only if $f$ is upper bounded on some set $B \subset G$ which is not null-finite in $X$ and whose closure $\bar{B}$ is contained in $G$.

Proof. The "only if" part is trivial. To prove the "if" part, assume that $f$ is upper bounded on some set $B \subset G$ such that $\bar{B} \subset G$ and $B$ is not null-finite in $X$. We need to check the continuity of $f$ at any point $c \in G$. Shifting the set $G$ and the function $f$ by $-c$, we may assume that $c=\theta$. Also we can replace the function $f$ by $f-f(\theta)$ and assume that $f(\theta)=0$. In this case the mid-point convexity of $f$ implies that $f\left(\frac{1}{2^{n}} x\right) \leq \frac{1}{2^{n}} f(x)$ for every $x \in G$ and $n \in \mathbb{N}$.

To derive a contradiction, suppose that the function $f$ is not continuous at $\theta$. Then there exists $\varepsilon>0$ such that $f(U) \not \subset(-\varepsilon, \varepsilon)$ for any open neighborhood $U \subset G$. For every $n \in \omega$ consider the neighborhood $U_{n}:=\{x \in$ $\left.G:\|x\| \leq \frac{1}{4^{n}}, x \in\left(\frac{1}{2^{n}} G\right) \cap\left(-\frac{1}{2^{n}} G\right)\right\}$ and find a point $x_{n} \in U_{n}$ such that $\left|f\left(x_{n}\right)\right| \geq \varepsilon$. Replacing $x_{n}$ by $-x_{n}$, if necessary, we can assume that $f\left(x_{n}\right) \geq \varepsilon$ (this follows from the inequality $\frac{1}{2}\left(f\left(x_{n}\right)+f\left(-x_{n}\right)\right) \geq f(\theta)=0$ ensured by the mid-point convexity of $f$ ).

Since $x_{n} \in \frac{1}{2^{n}} G$, for every $k \leq n$ the point $2^{k} x_{n}$ belongs to $G$ and has prenorm $\left\|2^{k} x_{n}\right\| \leq 2^{k}\left\|x_{n}\right\| \leq \frac{2^{n}}{4^{n}}=\frac{1}{2^{n}}$ in the metric linear space $(X,\|\cdot\|)$. This implies that $\left(2^{n} x_{n}\right)_{n \in \omega}$ is a null-sequence in $X$. Since the set $B$ is not null-finite, there exists $a \in X$ such that the set $\Omega:=\left\{n \in \mathbb{N}: a+2^{n} x_{n} \in B\right\}$ is infinite. Then $a \in \bar{B} \subset G$. Choose $k \in \mathbb{N}$ so large that $2^{-k+1} a \in-G$ (such number $k$ exists as $-G$ is a neighborhood of $\theta$ ).

Observe that for every number $n \in \Omega$ with $n>k$ the mid-point convexity of $f$ ensures that

$$
\begin{aligned}
2^{n-k} \varepsilon & \leq 2^{n-k} f\left(x_{n}\right) \leq f\left(2^{n-k} x_{n}\right)=f\left(\frac{-2^{-k+1} a}{2}+\frac{2^{-k+1} a+2^{n-k+1} x_{n}}{2}\right) \leq \\
& \leq \frac{1}{2} f\left(-2^{-k+1} a\right)+\frac{1}{2} f\left(2^{-k+1} a+2^{n-k+1} x_{n}\right) \leq \frac{1}{2} f\left(-2^{-k+1} a\right)+2^{-k} f\left(a+2^{n} x_{n}\right)
\end{aligned}
$$

and hence

$$
\sup f(B) \geq \sup _{n \in \Omega} f\left(a+2^{n} x_{n}\right) \geq \sup _{k<n \in \Omega}\left(2^{n} \varepsilon-2^{k-1} f\left(-2^{-k+1} a\right)\right)=\infty,
$$

which contradicts the upper boundedness of $f$ on $B$.

Theorem 11.1 implies the following generalization of the classical Bernstein-Doetsch Theorem [5], due to Mehdi [24]; see also a survey paper of Bingham and Ostaszewski [7].

Corollary 11.2. A mid-point-convex function $f: G \rightarrow \mathbb{R}$ defined on an open convex subset $G$ of a metric linear space $X$ is continuous if and only if $f$ is upper-bounded on some non-empty open subset of $G$. 
Combining Theorem 11.1 with Theorems 5.1 and 6.1 we obtain the following two continuity criteria, which answer the Problem of Baron and Ger [3, P239].

Corollary 11.3. A mid-point convex function $f: G \rightarrow \mathbb{R}$ defined on a convex subset $G$ of a complete metric linear space $X$ is continuous if and only if it is upper bounded on some uBP-set $B \subset G$ which is not Haar-meager in $X$.

Proof. Assume that $f$ is upper bounded on some uBP-set $B \subset G$ which is not Haar-meager in $X$.

Write the open set $G$ of $X$ as the union $U=\bigcup_{n \in \omega} F_{n}$ of closed subsets of $X$. By [9], the countable union of uBP Haar-meager sets is Haar-meager. Since the set $B=\bigcup_{n \in \omega} B \cap F_{n}$ is not Haar-meager, for some $n \in \mathbb{N}$ the subset $B \cap F_{n}$ of $B$ is not Haar-meager. By Theorem [5.1, $B \cap F_{n}$ is not null-finite. Since $\overline{B \cap F_{n}} \subset F_{n} \subset G$ and $f$ is upper bounded on $B \cap F_{n}$, we can apply Theorem 11.1 and conclude that the mid-point convex function $f$ is continuous.

Corollary 11.4. A mid-point convex function $f: G \rightarrow \mathbb{R}$ defined on a convex subset $G$ of a complete metric linear space $X$ is continuous if and only if it is upper bounded on some universally measurable set $B \subset G$ which is not Haar-null in $X$.

Proof. The proof of this corollary runs in exactly the same way as the proof of Corollary 11.3 and uses the well-known fact [8 that the countable union of universally measurable Haar-null sets in a complete metric group is Haar-null.

\section{Some Open Problems}

In this section we collect some open problems related to null-finite sets.

It is well-known that for a locally compact Polish group $X$, each Haar-meager set in $X$ can be enlarged to a Haar-meager $F_{\sigma}$-set and each Haar-null set in $X$ can be enlarged to a Haar-null $G_{\delta}$-set in $X$. Those "enlargement" results dramatically fail for non-locally compact Polish groups, see [13, [10]. We do not know what happens with null-finite sets in this respect.

Problem 12.1. Is each Borel null-finite subset $A$ of a (compact) Polish group $X$ contained in a null-finite set $B \subset X$ of low Borel complexity?

By [28 (resp. [12, 4.1.6]), each analytic Haar-null (resp. Haar-meager) set in a Polish group is contained in a Borel Haar-null (resp. Haar-meager) set. On the other hand, each non-locally compact Polish group contains a coanalytic Haar-null (resp. Haar-meager) set which cannot be enlarged to a Borel Haar-null (resp. Haar-meager) set, see [13] (resp. [10]).

Problem 12.2. Is each (co)analytic null-finite set $A$ in a Polish group $X$ contained in a Borel null-finite set?

Our next problem ask about the relation of the $\sigma$-ideal $\sigma \mathrm{NF}_{X}$ generated by null-finite sets to other known $\sigma$-ideals.

Problem 12.3. Let $X$ be a Polish group.

(1) Is $\sigma \overline{\mathrm{HN}}_{X} \subset \sigma \mathrm{NF}_{X}$ ?

(2) Is $\sigma \mathrm{NF}_{X}=\mathrm{HN}_{X} \cap \mathrm{HM}_{X}$ ?

The negative answer to both parts of Problem 12.3 would follow from the negative answer to the following concrete question.

Problem 12.4. Can the closed Haar-null set $\prod_{n=2}^{\infty} C_{n}^{*}$ in the group $X=\prod_{n=2}^{\infty} C_{n}$ be written as a countable union of Borel null-finite sets in $X$ ?

Problem 12.5. For an infinite Polish group $X$ and an ideal $\mathcal{I} \in\left\{\sigma \overline{\mathrm{NF}}_{X}, \sigma \mathrm{NF}_{X}\right\}$ evaluate the standard cardinal characteristics of $\mathcal{I}$ :

- $\operatorname{add}(\mathcal{I})=\min \{|\mathcal{J}|: \mathcal{J} \subset \mathcal{I}$ and $\bigcup \mathcal{J} \notin \mathcal{I}\}$

- $\operatorname{non}(\mathcal{I})=\min \{|A|: A \subset X$ and $A \notin \mathcal{I}\}$

- $\operatorname{cov}(\mathcal{I})=\min \{|\mathcal{J}|: \mathcal{J} \subset \mathcal{I}$ and $\bigcup \mathcal{J}=X\}$

- $\operatorname{cof}(\mathcal{I})=\min \{|\mathcal{J}|: \forall I \in \mathcal{I} \exists J \in \mathcal{J}(I \subset J)\}$.

The cardinal characteristics of the $\sigma$-ideals $\mathrm{HN}_{X}, \mathrm{HM}_{X}, \sigma \overline{\mathrm{HN}}_{X}$ on Polish groups are well-studied [4], 2] and play an important role in Modern Set Theory. 


\section{REFERENCES}

[1] A. Arhangel'skii, M. Tkachenko, Topological groups and related structures, Atlantis Studies in Mathematics, 1. Atlantis Press, Paris; World Scientific Publishing Co. Pte. Ltd., Hackensack, NJ, 2008.

[2] T. Banakh, Cardinal characteristics of the ideal of Haar null sets, Comment. Math. Univ. Carolin. 45:1 (2004), 119-137.

[3] K. Baron, R. Ger, Problem (P239), in: The 21st International Symposium on Functional Equations, August 6-13, 1983, Konolfingen, Switzerland, Aequationes Math. 26 (1984) 225-294.

[4] T. Bartoszynski, H. Judah, Set theory. On the structure of the real line, A K Peters, Ltd., Wellesley, MA, 1995.

[5] F. Bernstein, G. Doetsch, Zur Theorie der konvexen Funktionen, Math. Ann. 76:4 (1915) 514-526.

[6] N.H. Bingham, A.J. Ostaszewski, The Steinhaus-Weil property: its converse, Solecki amenability and subcontinuity, preprint (https://arxiv.org/abs/1607.00049).

[7] N.H. Bingham, A.J. Ostaszewski, Category-measure duality: convexity, mid-point convexity and Berz sublinearity, preprint (https://arxiv.org/abs/1607.05750).

[8] J.P.R. Christensen, On sets of Haar measure zero in abelian Polish groups, Israel J. Math. 13 (1972) $255-260$.

[9] U.B. Darji, On Haar meager sets, Topology Appl. 160 (2013) 2396-2400.

[10] M. Doležal, V. Vlasăk, Haar meager sets, their hulls, and relationship to compact sets, J. Math. Anal. Appl. 446:1 (2017) $852-863$.

[11] M. Doležal, V. Vlasăk, B. Vejnar, M. Rmoutil, Haar Meager Sets revisited, J. Math. Anal. Appl. 440:2 (2016) $922-939$.

[12] M. Elekes, D. Nagy, Haar null and Haar meager sets: a survey and new results, preprint (https://arxiv.org/abs/1508.02053).

[13] M. Elekes, Z. Vidnyánszky, Haar null sets without $G_{\delta}$ hulls, Israel J. Math. 209 (2015) 199-214.

[14] M. Fabian, P. Habala, P. Hajek, V. Montesinos, J. Pelant, V. Zizler, Functional analysis and infinite-dimensional geometry, Springer-Verlag, New York, 2001.

[15] P. Fischer, Z. Słodkowski, Christensen zero sets and measurable convex functions, Proc. Amer. Math. Soc. 79 (1980) $449-453$.

[16] B.R. Hunt, T. Sauer, J.A. Yorke, Prevalence: a translation-invariant "almost every" on infinite-dimensional spaces, Bull. Amer. Math. Soc. 27 (1992) 217-238.

[17] B.R. Hunt, T. Sauer, J.A. Yorke, Prevalence: an addendum, Bull. Amer. Math. Soc. 28 (1993) 306-307.

[18] E. Jabłońska, Some analogies between Haar meager sets and Haar null sets in abelian Polish groups, J. Math. Anal. Appl. 421 (2015) 1479-1486.

[19] A. Kechris, Classical Descriptive Set Theory, Springer-Verlag, New York, 1995.

[20] M. Kuczma, An introduction to the theory of functional equations and inequalities. Cauchy's Equation and Jensen's Inequality, PWN \& Uniwersytet Śląski w Katowicach, Warszawa-Kraków-Katowice, 1985.

[21] A. Kwela, Haar-smallest sets, (https://arxiv.org/abs/1711.09753).

[22] Ie. Lutsenko, I.V. Protasov, Sparse, thin and other subsets of groups, Internat. J. Algebra Comput. 19:4 (2009) 491-510.

[23] E. Matoušková, M. Zelený, A note on intersections of non-Haar null sets, Colloq. Math. 96 (2003) 1-4.

[24] M.R. Mehdi, On convex functions, J. London Math. Soc. 39 (1964) 321-326.

[25] B.J. Pettis, Remarks on a theorem of E. J. McShane, Proc. Amer. Math. Soc. 2 (1951) 166-171.

[26] S. Piccard, Sur les ensembles de distances des ensembles de points d'un espace Euclidien, Mem. Univ. Neuchâtel, vol. 13, Secrétariat Univ., Neuchâtel, 1939.

[27] H. Schaefer, Topological vector spaces, Springer-Verlag, New York-Berlin, 1971.

[28] S. Solecki, On Haar null sets, Fund. Math. 149 (1996) 205-210.

[29] H. Steinhaus, Sur les distances des points des ensembles de mesure positive, Fund. Math. 1 (1920) 99-104.

T. Banakh: Ivan Franko National University of Lviv (Ukraine) and Jan Kochanowski University in Kielce (Poland) E-mail address: t.o.banakh@gmail.com

E. Jabeońska: Department of Discrete Mathematics, Rzeszów University of Technology (Poland)

E-mail address: elizapie@prz.edu.pl 